\title{
Approximation d'un nombre réel par des nombres algébriques de degré donné
}

\author{
par \\ YAnn Bugeaud (Strasbourg) et Olivier Teulié (Talence)
}

1. Introduction. Dans ce travail, motivés par les récents travaux de Y. Bugeaud, G. Diaz, M. Laurent, D. Roy et M. Waldschmidt [2], [5]-[9], nous nous intéressons à l'approximation des nombres réels par des nombres algébriques, et en particulier par des entiers algébriques, problème qui, à ce jour, n'a été abordé que par H. Davenport \& W. M. Schmidt [4]. Afin d'énoncer les résultats connus, il convient d'introduire préalablement quelques notations et définitions.

Si $P(X)$ est un polynôme à coefficients entiers s'écrivant

$$
P(X)=a_{d} X^{d}+\ldots+a_{1} X+a_{0},
$$

on note $\mathrm{H}(P)$ sa hauteur naïve,

$$
\mathrm{H}(P)=\max _{0 \leq j \leq d}\left|a_{j}\right| .
$$

La hauteur du nombre algébrique $\alpha$ est, par définition, celle de son polynôme minimal sur $\mathbb{Z}$. En outre, si $\alpha$ est une racine du polynôme $P(X)$, un résultat de Gelfond (cf. par exemple [12], page 77) assure que

$$
e^{-d} \mathrm{H}(\alpha) \leq \mathrm{H}(P) \text {. }
$$

DÉfinition. Soient $n \geq 1$ un entier et $\xi$ un nombre complexe. On note $w_{n}(\xi)$ la borne supérieure des nombres réels $w$ pour lesquels il existe une infinité de polynômes $P$ à coefficients entiers, de degré majoré par $n$, vérifiant

$$
0<|P(\xi)| \leq \mathrm{H}(P)^{-w} .
$$

De même, on note $w_{n}^{*}(\xi)$ la borne supérieure des nombres réels $w^{*}$ pour lesquels il existe une infinité de nombres algébriques $\alpha$, de degré majoré par $n$, vérifiant

$$
0<|\xi-\alpha| \leq \mathrm{H}(\alpha)^{-w^{*}-1}
$$

2000 Mathematics Subject Classification: Primary 11J04; Secondary 11J68. 
Enfin, on note $w_{n}^{\prime}(\xi)$ la borne supérieure des nombres réels $w^{\prime}$ pour lesquels il existe une infinité d'entiers algébriques $\alpha$, de degré majoré par $n$, vérifiant

$$
0<|\xi-\alpha| \leq \mathrm{H}(\alpha)^{-w^{\prime}} \text {. }
$$

Soient $n \geq 1$ un entier et $\xi$ un nombre réel. Il découle du théorème de Minkowski (cf. par exemple [11], Theorem 2C, page 33) que $w_{n}(\xi) \geq$ $n$ si $\xi$ n'est pas algébrique de degré $\leq n$. En outre, on voit facilement que $w_{n}(\xi) \geq w_{n}^{*}(\xi)$ (cf. [11], page 279), mais par contre il semble difficile de minorer non trivialement $w_{n}^{*}(\xi)$ en fonction de $w_{n}(\xi)$. Le problème de l'évaluation précise des nombres $w_{n}^{*}(\xi)$ et $w_{n}^{\prime}(\xi)$ est ouvert, et l'énoncé généralement conjecturé (cf. [10]) est le suivant.

Conjecture. Soient $n \geq 1$ un entier et $\xi$ un nombre réel qui n'est pas algébrique de degré $\leq n$. Soit $\xi^{\prime}$ un nombre réel qui n'est ni algébrique de degré $<n$, ni entier algébrique de degré $n$. Alors on a

$$
w_{n}^{*}(\xi) \geq n \quad \text { et } \quad w_{n}^{\prime}\left(\xi^{\prime}\right) \geq n .
$$

Le premier résultat dans cette direction a été obtenu par E. Wirsing en $1961[14]$.

ThÉORÈme 1 (E. Wirsing). Soient $n \geq 1$ un entier et $\xi$ un nombre réel qui n'est pas algébrique de degré $\leq n$. On a alors

et

$$
\begin{gathered}
w_{n}^{*}(\xi) \geq \frac{w_{n}(\xi)}{w_{n}(\xi)-n+1} \\
w_{n}^{*}(\xi) \geq \frac{n+2}{4}+\frac{\sqrt{n^{2}+4 n-4}}{4} .
\end{gathered}
$$

L'unique amélioration actuellement connue du Théorème 1 est due à V. Bernik \& K. Tishchenko [1], qui, grâce à une remarque astucieuse, sont parvenus à obtenir le résultat suivant.

ThÉorème 2 (V. Bernik \& K. Tishchenko). Sous les hypothèses du Théorème 1 , on $a$

$$
w_{n}^{*}(\xi) \geq \frac{n}{4}+\frac{\sqrt{n^{2}+16 n-8}}{4} .
$$

La démonstration du Théorème 2, ainsi qu'une nouvelle preuve de l'estimation (1), se trouve dans le survol [3].

En ce qui concerne l'approximation par des entiers algébriques, le seul résultat connu à ce jour est dû à $H$. Davenport \& W. M. Schmidt [4]. Son énoncé fait intervenir la suite $\left(\nu_{n}\right)_{n \geq 2}$ définie par :

$$
\nu_{2}=2, \quad \nu_{3}=\frac{3+\sqrt{5}}{2}, \quad \nu_{4}=3, \quad \nu_{n}=\left[\frac{n+1}{2}\right] \quad \text { pour } n \geq 5,
$$

qui apparaît également dans notre Théorème 5 . 
Théorème 3 (H. Davenport \& W. M. Schmidt). Si $n$ est un entier $\geq 5$ (resp. si $n=3$ ou 4 , si $n=2$ ) et si $\xi$ est un réel non algébrique de degré $\leq(n-1) / 2($ resp. $\leq 2, \leq 1)$, alors

$$
w_{n}^{\prime}(\xi) \geq \nu_{n}
$$

Les travaux récents de Y. Bugeaud, G. Diaz, M. Laurent, D. Roy et M. Waldschmidt [2], [5]-[9] abordent ces problèmes d'approximation en reprenant certaines idées de E. Wirsing, mais en utilisant la mesure de Mahler au lieu de la hauteur naïve. Cela apporte une flexibilité permettant d'obtenir des généralisations du Théorème 1 - à ceci près que la minoration de $w_{n}^{*}(\xi)$ est moins bonne qu'en (2) - , ainsi que de nouvelles informations sur la taille et le degré des bons approximants des nombres réels. Comme corollaire de leur résultat principal, M. Laurent \& D. Roy [7] ont ainsi montré que si $\xi$ est un nombre réel transcendant et si $n \geq 26$ est un entier, alors il existe une infinité de nombres algébriques $\alpha$ vérifiant

$$
0.001 n \leq \operatorname{deg}(\alpha) \leq n \quad \text { et } \quad|\xi-\alpha| \leq \mathrm{H}(\alpha)^{-n / 1000} .
$$

Dans le présent travail, nous parvenons à considérablement améliorer (4), en prouvant (cf. Théorème 5) que l'on peut en fait obtenir le même énoncé avec $n / 2$ au lieu de $n / 1000$ et, surtout, le degré des approximants $\alpha$ exactement égal à $n$. Nous montrons également (cf. Théorème 5) qu'un tel résultat reste valable si l'on demande en plus aux $\alpha$ d'être des entiers algébriques.

La méthode que nous suivons est celle de H. Davenport \& W. M. Schmidt [4]; elle diffère notablement de celle de E. Wirsing, laquelle, basée sur le théorème de Minkowski, ne permet pas de traiter l'approximation par des entiers algébriques. Elle consiste entre autres à construire une suite de polynômes dont on contrôle les hauteurs et qui, au contraire de leurs dérivées, prennent une valeur petite en $\xi$, le nombre réel que l'on souhaite approcher. L'ingrédient nouveau est l'observation que, grâce au critère d'Eisenstein, nous pouvons supposer ces polynômes irréductibles, et leur imposer des contraintes variées.

Notre article est organisé de la manière suivante. La partie 2 est consacrée à des rappels de géométrie des nombres, qui nous sont utiles dans la démonstration de notre résultat principal, le Théorème 4, présentée dans la partie 3 . Du Théorème 4 découlent de nombreux corollaires, dont les énoncés détaillés figurent dans la quatrième et dernière partie.

Remerciements. Les résultats du présent travail ont été obtenus indépendamment par les deux auteurs, qui tiennent à remercier Bernard de Mathan pour ses nombreuses et pertinentes observations, ainsi que l'arbitre pour ses remarques judicieuses concernant la présentation de l'article. 
2. Minima successifs d'un convexe de $\mathbb{R}^{n}$. On définit pour un convexe $K$ de $\mathbb{R}^{n}$ et un entier $p \leq n$ le $p$-ième des minima successifs comme le plus petit réel $\tau_{p}$ tel que le convexe $\tau_{p} K$ contienne au moins $p$ points à coordonnées entières, linéairement indépendantes. Ainsi, si le convexe $K$ est défini par un système d'inéquations

$$
\begin{aligned}
\left|f_{1}\left(x_{1}, \ldots, x_{n}\right)\right| & \leq 1, \\
& \vdots \\
\left|f_{n}\left(x_{1}, \ldots, x_{n}\right)\right| & \leq 1,
\end{aligned}
$$

où les $f_{i}$ sont des polynômes linéaires homogènes, alors le $p$-ième des minima successifs de $K$ est le plus petit réel $\tau_{p}$ tel que le système

$$
\begin{aligned}
\left|f_{1}\left(x_{1}, \ldots, x_{n}\right)\right| & \leq \tau_{p}, \\
& \vdots \\
\left|f_{n}\left(x_{1}, \ldots, x_{n}\right)\right| & \leq \tau_{p}
\end{aligned}
$$

possède $p$ solutions entières linéairement indépendantes.

2.1. La méthode de H. Davenport \& W. M. Schmidt. Soit $n \geq 2$ un entier et soit $\xi$ un nombre réel qui n'est pas algébrique de degré $\leq(n-1) / 2$. D'après les travaux de H. Davenport \& W. M. Schmidt [4], il existe une infinité de réels positifs $Y$ arbitrairement grands tels que le $n$-ième des minima successifs du convexe défini par

$$
\left\{\begin{array}{l}
\left|x_{n-1} \xi^{n-1}+\ldots+x_{1} \xi+x_{0}\right| \leq Y^{-n+1} \\
\left|x_{m}\right| \leq Y \quad(m=1, \ldots, n-1)
\end{array}\right.
$$

est majoré, à une constante multiplicative près, par $Y^{\varrho_{n}}$, avec $\varrho_{n}=n / \nu_{n}-1$, où la suite $\left(\nu_{n}\right)_{n>2}$ est définie dans l'introduction. Ainsi, pour de tels $Y$, il existe une constante $c$, ne dépendant que de $\xi$ et de $n$, et $n$ polynômes de degré au plus $n-1$, linéairement indépendants et à coefficients dans $\mathbb{Z}$

$$
P_{i}(X)=x_{n-1}^{(i)} X^{n-1}+\ldots+x_{1}^{(i)} X+x_{0}^{(i)} \quad(i=1, \ldots, n),
$$

tels que, pour $1 \leq i \leq n$,

$$
\begin{aligned}
\left|P_{i}(\xi)\right| & \leq c Y^{1+\varrho_{n}-n}, \\
\mathrm{H}\left(P_{i}\right) & \leq c Y^{1+\varrho_{n}} .
\end{aligned}
$$

Grâce à cela, H. Davenport et W. M. Schmidt ont prouvé le Théorème 3 , énoncé dans l'introduction.

2.2. Une autre majoration du n-ième des minima successifs. Dans tout ce qui suit, la constante numérique sous-entendue dans $\ll$ (resp. dans $\ll_{\varepsilon}$ ) ne dépend que de $n$ et de $\xi$ (resp. que de $n, \xi$ et $\varepsilon$ ). 
Outre l'estimation de H. Davenport \& W. M. Schmidt, on peut également donner une majoration du $n$-ième des minima successifs du convexe $\mathcal{K}(Y)$ en fonction de $w_{n-1}(\xi)$ :

Lemme 1. Soient $\varepsilon>0, n \geq 2$ un entier et $\xi$ un nombre réel non algébrique de degré au plus $n-1$. Alors le n-ième des minima successifs $\tau_{n}(Y)$ de $\mathcal{K}(Y)$ vérifie

$$
\tau_{n}(Y) \ll_{\varepsilon} Y^{(n-1) \frac{w_{n-1}(\xi)-n+1}{1+w_{n-1}(\xi)}+\varepsilon} .
$$

Démonstration. Soit $Y>0$ un entier et notons $\tau_{1}(Y)$ (resp. $\tau_{n}(Y)$ ) le premier (resp. dernier) des minima successifs de $\mathcal{K}(Y)$. Il existe donc un polynôme $P$ à coefficients entiers, de degré au plus $n-1$, tel que

$$
|P(\xi)| \leq \tau_{1}(Y) Y^{1-n} \quad \text { et } \quad \mathrm{H}(P) \ll \tau_{1}(Y) Y .
$$

D'après la définition de $w_{n-1}(\xi)$, comme $\varepsilon$ est un nombre réel strictement positif, on a pour tout polynôme $Q$ à coefficients entiers, non nul et de degré au plus $n-1$,

$$
|Q(\xi)| \gg_{\varepsilon} \mathrm{H}(Q)^{-w_{n-1}(\xi)-\varepsilon} .
$$

On obtient donc,

$$
\left(\tau_{1}(Y) Y\right)^{-w_{n-1}(\xi)-\varepsilon} \ll_{\varepsilon} \tau_{1}(Y) Y^{1-n},
$$

c'est-à-dire

$$
\tau_{1}(Y) \gg_{\varepsilon} Y^{-\frac{w_{n-1}(\xi)-n+1}{1+w_{n-1}(\xi)}-\varepsilon} .
$$

De la majoration $\tau_{1}(Y) \ldots \tau_{n}(Y) \ll 1$ et de la minoration de $\tau_{1}(Y)$ on déduit alors que

$$
\tau_{n}(Y) \ll_{\varepsilon} Y^{(n-1) \frac{w_{n-1}(\xi)-n+1}{1+w_{n-1}(\xi)}+\varepsilon} .
$$

Remarque. Cette majoration du $n$-ième des minima successifs ne donne pas un exposant indépendant de $\xi$, au contraire de celle de H. Davenport $\&$ W. M. Schmidt. Elle est triviale pour les réels $\xi$ vérifiant $w_{n-1}(\xi)=\infty$ : de tels nombres existent et forment un ensemble non dénombrable (cf. par exemple [12]).

3. Résultat principal. L'objet de cette partie est la démonstration du résultat suivant, qui constitue notre théorème principal.

ThÉORÈme 4 . Soient $\xi$ un nombre réel et $n \geq 2$ un entier. On suppose qu'il existe un réel $\varrho_{n}$ et des nombres $Y$ arbitrairement grands pour lesquels le $n$-ième des minima successifs du convexe $\mathcal{K}(Y)$ vérifie

$$
\tau_{n}(Y) \ll Y^{\varrho_{n}} .
$$


Alors il existe une infinité de nombres algébriques (resp. d'entiers algébriques), $\alpha$ réels de degré exactement $n-1$ (resp. $n$ ) tels que

$$
|\xi-\alpha| \ll\left(\frac{\mathrm{H}(\alpha)}{\log \mathrm{H}(\alpha)}\right)^{-n /\left(1+\varrho_{n}\right)} .
$$

Dé mo n stration. D'après la définition de $\tau_{n}(Y)$, il existe $n$ polynômes $\left(P_{i}\right)_{1 \leq i \leq n}$ à coefficients entiers, de degré au plus $n-1$, linéairement indépendants vérifiant (5) et (6). Comme les polynômes sont linéairement indépendants, il existe un indice $i$ tel que $x_{0}^{(i)}$ soit non nul, et l'on peut supposer que $i=1$. On note alors $p$ le plus petit nombre premier ne divisant pas $x_{0}^{(1)}$. D'après le théorème des nombres premiers, on a

$$
p \ll \log x_{0}^{(1)} .
$$

On va alors construire un polynôme $p$-d'Eisenstein, dont la valeur est petite en $\xi$ et dont la valeur de la dérivée est grande en $\xi$.

Soit $d \geq n-1$ un entier et considérons le système de $n$ équations à $n$ inconnues $\left(\theta_{i}\right)_{1 \leq i \leq n}$ suivant :

$$
\begin{aligned}
& \xi^{d}+p\left(\theta_{1} P_{1}(\xi)+\ldots+\theta_{n} P_{n}(\xi)\right)=p(n+1) c Y^{\varrho_{n}-n+1}, \\
& d \xi^{d-1}+p\left(\theta_{1} P_{1}^{\prime}(\xi)+\ldots+\theta_{n} P_{n}^{\prime}(\xi)\right)=p Y^{1+\varrho_{n}}+p \sum_{1 \leq i \leq n}\left|P_{i}^{\prime}(\xi)\right|, \\
& \theta_{1} x_{m}^{(1)}+\ldots+\theta_{n} x_{m}^{(n)}=0 \quad(m=2, \ldots, n-1) .
\end{aligned}
$$

Comme les $P_{i}$ sont linéairement indépendants, il s'agit d'un système de Cramer, qui admet donc une unique solution réelle. On approche le $n$-uplet solution $\left(\theta_{1}, \ldots, \theta_{n}\right)$ par un $n$-uplet entier $\left(t_{1}, \ldots, t_{n}\right)$ tel que $\left|\theta_{i}-t_{i}\right| \leq 1$ et on pose

$$
x_{m}=t_{1} x_{m}^{(1)}+\ldots+t_{n} x_{m}^{(n)} \quad \text { pour } m=0, \ldots, n-1 .
$$

On considère alors le polynôme suivant :

$$
\begin{aligned}
P(X) & =X^{d}+p\left(x_{n-1} X^{n-1}+\ldots+x_{1} X+x_{0}\right) \\
& =X^{d}+p\left(t_{1} P_{1}(X)+\ldots+t_{n} P_{n}(X)\right) .
\end{aligned}
$$

Pour qu'il soit $p$-d'Eisenstein, il suffit de vérifier que son coefficient constant, en l'occurrence $p\left(t_{1} x_{0}^{(1)}+\ldots+t_{n} x_{0}^{(n)}\right)$, n'est pas divisible par $p^{2}$ car son coefficient dominant est congru à 1 modulo $p$. On fixe alors un $(n-1)$-uplet $\left(t_{2}, \ldots, t_{n}\right)$ et il reste deux choix pour $t_{1}$, que l'on peut noter $t_{1,0}$ et $t_{1,1}=t_{1,0}+1$. Comme $p$ ne divise pas $x_{0}^{(1)}$, l'un des deux entiers $t_{1,0} x_{0}^{(1)}+\ldots+t_{n} x_{0}^{(n)}$ ou $t_{1,1} x_{0}^{(1)}+\ldots+t_{n} x_{0}^{(n)}$ n'est pas divisible par $p$; on peut par conséquent choisir $t_{1}$ de sorte que le polynôme $P$ soit $p$-d'Eisenstein. 
En outre, le polynôme $P$ vérifie la propriété suivante :

$$
P(\xi)=\xi^{d}+p\left(t_{1} P_{1}(\xi)+\ldots+t_{n} P_{n}(\xi)\right),
$$

donc, d'après (5) et la première équation du système, on a

$$
0<P(\xi) \ll p Y^{\varrho_{n}-n+1} .
$$

D'autre part,

$$
P^{\prime}(\xi)=d \xi^{d-1}+p\left(t_{1} P_{1}^{\prime}(\xi)+\ldots+t_{n} P_{n}^{\prime}(\xi)\right),
$$

donc, d'après (6) et la deuxième équation du système, on a

$$
P^{\prime}(\xi) \ll p Y^{1+\varrho_{n}},
$$

et

$$
P^{\prime}(\xi) \geq p Y^{1+\varrho_{n}} .
$$

Enfin, grâce à (6) et à la dernière série d'équations du système, on obtient

$$
\left|x_{m}\right| \ll Y^{1+\varrho_{n}} \quad \text { pour } m=2, \ldots, n-1 ;
$$

donc, compte tenu de (9) et (11),

$$
\left|x_{1}\right| \ll Y^{1+\varrho_{n}} .
$$

Comme, de (8), (11) et (12), découle

$$
\left|x_{0}\right| \ll Y^{1+\varrho_{n}},
$$

on obtient finalement

$$
\mathrm{H}(P) \ll p Y^{1+\varrho_{n}} .
$$

Il existe donc une racine réelle $\alpha$ de $P$, c'est-à-dire un entier algébrique de degré $n$, telle que

$$
0<|\xi-\alpha| \ll\left|\frac{P(\xi)}{P^{\prime}(\xi)}\right| \ll Y^{-n},
$$

car $P^{\prime}(x) \gg p Y^{1+\varrho_{n}}$ pour $|x-\xi| \ll 1$. Or d'après (7), (13) et (14),

$$
\mathrm{H}(\alpha) \ll Y^{1+\varrho_{n}} \log Y,
$$

donc finalement,

$$
0<|\xi-\alpha| \ll\left(\frac{\mathrm{H}(\alpha)}{\log \mathrm{H}(\alpha)}\right)^{-n /\left(1+\varrho_{n}\right)} .
$$

Pour conclure, il suffit de choisir $d=n-1$ (resp. $d=n$ ) dans le cas de l'approximation par des nombres (resp. par des entiers) algébriques. 
Remarque. En prenant $d=n+1$, on démontre qu'il existe une infinité d'entiers algébriques de trace nulle $\alpha$, de degré $n+1$, tels que

$$
0<|\xi-\alpha| \ll\left(\frac{\mathrm{H}(\alpha)}{\log \mathrm{H}(\alpha)}\right)^{-n /\left(1+\varrho_{n}\right)} .
$$

Le majorant étant toujours le même, on voit que le paramètre qui entre en jeu est le nombre de coefficients libres du polynôme minimal de $\alpha$.

\section{Application à l'approximation des nombres réels}

4.1. Un complément au Théorème 3. D'après les travaux de H. Davenport \& W. M. Schmidt, on peut appliquer le Théorème 4 à la suite $\varrho_{n}=n / \nu_{n}-1$, où l'on rappelle que $\left(\nu_{n}\right)_{n \geq 2}$, définie dans l'introduction, est donnée par $\nu_{2}=2, \nu_{3}=(3+\sqrt{5}) / 2, \nu_{4}=3$ et $\nu_{n}=[(n+1) / 2]$, pour $n \geq 5$. On obtient alors le résultat suivant :

ThÉORÈme 5. Soient $n \geq 5$ (resp. $n=3$ ou $4, n=2$ ) un entier et $\xi$ un nombre réel, non algébrique de degré au plus $(n-1) / 2$ (resp. au plus 2 , au plus 1). Alors il existe une infinité de nombres algébriques (resp. d'entiers algébriques) $\alpha$ réels de degré exactement $n-1$ (resp. $n$ ) tels que

$$
0<|\xi-\alpha| \ll \mathrm{H}(\alpha)^{-\nu_{n}}(\log \mathrm{H}(\alpha))^{\nu_{n}} .
$$

4.2. Approximation presque sûre et approximation des nombres algébriques. Pour tout réel $\varepsilon>0$, on peut, d'après le Lemme 1, appliquer le Théorème 4 avec

$$
\varrho_{n}=(n-1)\left(1-\frac{n}{1+w_{n-1}(\xi)}\right)+\varepsilon,
$$

afin de minorer $w_{n}^{\prime}(\xi)$ en fonction de $w_{n}(\xi)$.

THÉORÈme 6 . Soit $n \geq 3$ un entier. Soit $\xi$ un nombre réel qui n'est pas algébrique de degré $\leq n-1$. Alors

$$
w_{n}^{\prime}(\xi) \geq \frac{w_{n-1}(\xi)+1}{w_{n-1}(\xi)-n+2} .
$$

En outre, pour tout réel $\varepsilon>0$ fixé, il existe une infinité d'entiers algébriques $\alpha$ réels de degré exactement $n$ tels que

$$
0<|\xi-\alpha|<\mathrm{H}(\alpha)^{-\frac{w_{n-1}(\xi)+1}{w_{n-1}(\xi)-n+2}+\varepsilon} .
$$

Résolvant une conjecture de Mahler, V. G. Sprindžuk [13] a démontré que, pour presque tout nombre réel transcendant $\xi-$ au sens de la mesure de Lebesgue - on a $w_{n-1}(\xi)=n-1$. Du Théorème 6 découle alors le corollaire suivant. 
Corollaire 1. Soient $\varepsilon>0$ un réel et $n \geq 2$ un entier. Pour presque tout nombre réel $\xi$, il existe une infinité d'entiers algébriques $\alpha$ réels de degré exactement $n$ tels que

$$
0<|\xi-\alpha|<\mathrm{H}(\alpha)^{-n+\varepsilon} .
$$

En particulier, nous avons $w_{n}^{\prime}(\xi)=n$ pour presque tout $\xi$.

Concernant l'approximation des nombres réels algébriques par des nombres algébriques de plus petit degré, W. M. Schmidt a montré [10], comme conséquence de son célèbre théorème du sous-espace, que si $\xi$ est un nombre réel de degré $d$, alors, pour tout entier $n \leq d-1$, on a $w_{n}(\xi)=n$. Grâce au Théorème 6 , on retrouve le corollaire suivant qui se déduit aisément des Théorèmes $7 \mathrm{~J}$ et $7 \mathrm{~K}$ de [10].

Corollaire 2. Soient $\varepsilon>0$ un réel et $n \geq 2$ un entier. Soit $\xi$ un nombre réel algébrique de degré $\geq n$, et qui n'est pas un entier algébrique de degré $n$. Il existe alors une infinité d'entiers algébriques a réels de degré exactement $n$ tels que

$$
0<|\xi-\alpha|<\mathrm{H}(\alpha)^{-n+\varepsilon} .
$$

En particulier, nous avons $w_{n}^{\prime}(\xi)=n$.

\section{Références bibliographiques}

[1] V. Bernik and K. Tishchenko, Integral polynomials with an overfall of the coefficient values and Wirsing's problem, Dokl. Akad. Nauk Belarusi 37 (1993), 9-11.

[2] Y. Bugeaud, Approximation par des nombres algébriques, J. Number Theory, à paraître.

[3] - On the approximation by algebraic numbers with bounded degree, Proceedings of the Number Theory Conference held in Graz, à paraître.

[4] H. Davenport and W. M. Schmidt, Approximation to real numbers by algebraic integers, Acta Arith. 15 (1969), 393-416.

[5] G. Diaz, Une nouvelle propriété d'approximation diophantienne, C. R. Acad. Sci. Paris 324 (1997), 969-972.

[6] M. Laurent and D. Roy, Criteria of algebraic independence with multiplicities and interpolation determinants, Trans. Amer. Math. Soc. 351 (1999), 1845-1870.

[7] -, -, Sur l'approximation algébrique en degré de transcendance un, Ann. Inst. Fourier (Grenoble) 49 (1999), 27-55.

[8] D. Roy et M. Waldschmidt, Approximation diophantienne et indépendance algébrique de logarithmes, Ann. Sci. École Norm. Sup. 30 (1997), 753-796.

[9] -, - Simultaneous approximation and algebraic independence, Ramanujan Math. J. 1 (1997), 379-430.

[10] W. M. Schmidt, Approximation to Algebraic Numbers, Monograph. Enseign. Math. 19, Univ. de Genève, 1971.

[11] —, Diophantine Approximation, Lecture Notes in Math. 785, Springer, Berlin, 1980.

[12] T. Schneider, Introduction aux nombres transcendants, Gauthier-Villars, Paris, 1959. 
[13] V. G. Sprindžuk, Mahler's Problem in Metric Number Theory, Transl. Math. Monographs 25, Amer. Math. Soc., Providence, R.I., 1969.

[14] E. Wirsing, Approximation mit algebraischen Zahlen beschränkten Grades, J. Reine Angew. Math. 206 (1961), 67-77.

Université Louis Pasteur

U. F. R. de mathématiques

7 , rue René Descartes

67084 Strasbourg Cedex, France

E-mail: bugeaud@math.u-strasbg.fr
Université Bordeaux 1

U. F. R. Mathématiques-Informatique 351, cours de la Libération 33405 Talence Cedex, France E-mail: teulie@math.u-bordeaux.fr 